\title{
Estrategias de Trabajo Autónomo en Estudiantes Universitarios Noveles de Educación
}

\section{Individual Learning Strategies in Novels Students of Education}

\author{
$\mathrm{M}^{\mathrm{a}}$ del Carmen Pegalajar * \\ Universidad de Jaén, España
}

\begin{abstract}
El nuevo espacio creado en Educación Superior exige el desarrollo de nuevos roles y responsabilidades en el estudiante ante el proceso de enseñanza-aprendizaje, lo que le lleva la práctica de estrategias de aprendizaje autónomo y autorregulado en entornos de trabajo flexibles, fomentando su participación activa. Esta investigación examina el desarrollo de estrategias de trabajo autónomo en estudiantes noveles de Educación de la Universidad de Jaén $(n=407)$. Basados en una metodología descriptiva, se lleva a cabo la recogida de datos mediante el "Cuestionario de Estrategias de Trabajo Autónomo" a través de la técnica de la encuesta. Los resultados demuestran cómo las estrategias más desarrolladas por los estudiantes se relacionan con la preparación de exámenes y el trabajo intelectual del estudiante sobre el contenido de las asignaturas incluidas en los planes de estudio, siendo la estrategia de ampliación la que ha obtenido peores resultados. Además, los estudiantes más jóvenes ponen en práctica mejores estrategias de aprendizaje autónomo, lo que facilita la planificación del quehacer docente. Dicho análisis permite al profesorado universitario conocer las estrategias y habilidades de que dispone el estudiante para, de este modo, adaptar el proceso de enseñanzaaprendizaje a dichas circunstancias. Por su parte, el estudiante universitario puede adecuar sus preferencias de aprendizaje al perfil académico de las titulaciones de Educación, así como también supone un factor que propicia la mejora de las acciones formativas para los estudiantes de nuevo ingreso.
\end{abstract}

Descriptores: Aprendizaje activo; Estudiante; Enseñanza superior; Evaluación del estudiante.

The new space created in Higher Education requires the development of new roles and responsibilities in the student before the teaching-learning process, which leads to the practice of self-regulated and self-regulated learning strategies in flexible work environments, encouraging their active participation. This research examines the development of individual learning strategies in novel students of Education at the University of Jaen $(n=407)$. Based on a descriptive methodology, the data collection is carried out through the "Individual Learning Strategies Questionnaire", through the survey technique. The results show how the strategies most developed by the students are related to the preparation of exams and the intellectual work of the student on the content of the subjects included in the curricula, with the expansion strategy having the worst results. In addition, the youngest students put into practice better strategies of autonomous learning, which facilitates the planning of the teaching task. This analysis allows university professors to know the strategies and skills available to the student to, in this way, adapt the teaching-learning process to these circumstances. On the other hand, the university student can adapt their learning preferences to the academic profile of the Education degrees, as well as being a factor that favors the improvement of the training actions for new students.

Keywords: Activity learning; Student; Higher education; Student evaluation.

*Contacto: mcpegala@ujaen.es

ISSN: $1696-4713$

www.rinace.net/reice/

revistas.uam.es/reice
Recibido: $\quad 15$ de junio 2019

$1^{\text {a }}$ Evaluación: 20 de julio 2019

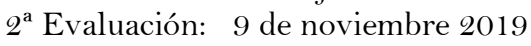

Aceptado: 28 de noviembre 2019 


\section{Introducción}

La transformación del actual escenario universitario gracias al proceso de convergencia europea ha supuesto la revisión de los modelos de docencia tradicionales, centrados en la adquisición de conocimientos teóricos por parte de los estudiantes de Educación Superior. Así pues, se ha pasado de la "universidad del enseñar" a la "universidad del aprender" (Tejada y Ruiz, 2016), desde una concepción del aprendizaje constructivista, basada en el conocimiento significativo y la autonomía del estudiante, quién conoce sus propios procesos cognitivos y controla su aprendizaje (Olmedo, 2013).

El nuevo espacio creado en Educación Superior apuesta por el desarrollo de estrategias que fomenten la participación activa del estudiante y su aprendizaje autónomo y autorregulado en entornos de trabajo más flexibles (Kramarski y Michalski, 2009, Rezende et al., 2014). Promueve el desarrollo de habilidades, destrezas y comportamientos que el estudiante debe demostrar en el desempeño de su trabajo, potenciando la interdisciplinariedad, el trabajo en grupo, la búsqueda y selección de información, la construcción del propio aprendizaje, la implicación de la persona en proyectos y la adquisición de capacidades personales (Imaz, 2015).

Gargallo, Suárez y Pérez (2009) definen las estrategias de aprendizaje como "un conjunto organizado, consciente e intencional de lo que hace el aprendiz para lograr con eficacia un objetivo de aprendizaje en un contexto social dado” (p.2). Éstas tienen un alto grado de complejidad, al ser procedimientos internos fundamentalmente de carácter cognitivo (Martínez y Bonachea, 2011); implican todo tipo de pensamientos, acciones, comportamientos, creencias y emociones que permiten y apoyan la adquisición y recuperación de la información y las maneras de relacionarla con el conocimiento previo (Muñoz, 2005).

Según López (2010), las estrategias de aprendizaje autónomo para el estudiante universitario involucran una serie de variables, entre las que se incluyen:

- Ampliación, relacionadas con la búsqueda y elaboración de materiales, así como su participación en el desarrollo de actividades complementarias sobre el material propuesto por el profesor.

- Colaboración, vinculada con la implicación del estudiante en el desarrollo de tareas grupales y que impliquen el trabajo compartido con otros compañeros.

- Conceptualización, relacionada con el trabajo intelectual del estudiante sobre el contenido a desarrollar en cada una de las asignaturas: elaboración de esquemas, resúmenes, mapas conceptuales, etc.

- Planificación de tiempos y programación de las tareas a realizar, tanto para el estudio como para la realización de trabajos. Asimismo, también contempla alguna variable vinculada a la evaluación de los procedimientos de aprendizaje.

- Preparación de exámenes, la cual incluye variables relacionadas con el estudio para la evaluación, especialmente, la selección de puntos importantes y actividades de repaso.

- Participación del alumno en el proceso de enseñanza-aprendizaje, vinculada al análisis del grado de asistencia a clase, aclaración de dudas, participación en el aula o asistencia a tutorías. 
Así pues, este nuevo paradigma educativo exige el desarrollo de nuevos roles y responsabilidades en el estudiante, llevándole a perfeccionar o modificar determinadas competencias, creencias o ideas acerca de la enseñanza universitaria y de los estudios que realiza (Fernández-Molina, González y Del Molino, 2011). Harrington y Parker (2013) apuestan por desarrollar un currículo que capacite a los estudiantes para el desarrollo de habilidades de autorregulación, colaboración y reflexión en contextos de aprendizaje auténticos; la opción de un aprendizaje más reflexivo, responsable, autónomo y cooperativo facilita la construcción significativa y el desarrollo de competencias profesionales entre los estudiantes de Educación Superior (Biggs, 2010; Coll, Mauri y Rochera, 2012; Domingo y Gómez, 2014).

Ante este contexto, resulta de utilidad conocer los estilos y las estrategias de aprendizaje del estudiante universitario, comprobando si éstos se ajustan a los requerimientos del Espacio Europeo de Educación Superior (Cuadrado, Monroy y Montaño, 2011) para, de este modo, mejorar la práctica educativa y lograr un aprendizaje integral en el estudiante universitario (Hidalgo y Murillo, 2017).

El interés por conocer la preferencia de los estudiantes universitarios hacia el aprendizaje constituye un tema de enorme interés en los últimos años (Alkahasawneh, 2013; Hallin, 2014; William, Brown y Etherington, 2013). En este sentido, Freiberg, Ledesma y Fernández (2017) describen cómo su análisis permite:

- Modificar las prácticas pedagógicas al profesorado universitario, ajustando sus recursos al perfil académico del estudiante.

- Mejorar los planes de estudio de la institución universitaria, para reforzar la articulación de contenidos teóricos y prácticos.

- Adaptar las preferencias de aprendizaje de los estudiantes a las características de los perfiles académicos de cada titulación, lo cual repercute en la eficacia de las elecciones de especialización del estudiante universitario.

Además, Bahamón y colaboradores (2013) o Mehrdad y Ahghar (2012) sostienen cómo el análisis de las estrategias de aprendizaje permite la obtención de información para posibilitar al profesorado la adaptación de sus modalidades de enseñanza y, por otro lado, el desarrollo de estrategias en los estudiantes para facilitar la incorporación de contenidos académicos específicos. Tal y como exponen Aguado y colaboradores (2017) es importante entender los factores que facilitan y explican el desempeño académico de los estudiantes en Educación Superior; ello supone la descripción del perfil académico del alumno, con importantes implicaciones para la institución universitaria y, más concretamente, para el profesor de Educación Superior, en un intento por ofrecer al estudiante los conocimientos, habilidades y actitudes necesarios para hacer formar a profesionales competentes.

De este modo, el problema de investigación al que se da respuesta en este trabajo queda formulado mediante el siguiente interrogante: ${ }_{\complement}$ Cuáles son las estrategias de trabajo autónomo que pone en práctica el estudiante novel de la Universidad de Jaén adscrito a los Grados en Educación? 


\section{Revisión de la literatura}

La investigación propuesta por Garrote, Garrote y Jiménez (2016) revela cómo la estrategia de aprendizaje más utilizada por los estudiantes de Grado es la de elaboración, la cual le permite aplicar conocimientos anteriores a nuevas situaciones para la resolución de problemas, toma de decisiones, evaluaciones críticas, etc. Además, este estudio destaca como la estrategia menos empleada es la de auto-interrogación, teniendo en cuenta la autoevaluación del propio estudiante para centrarse en el contenido de la materia, valorar su nivel de comprensión y cuestionar la veracidad de lo aprendido.

Por su parte, García-Valcárcel y Tejedor (2017) muestran cómo la cantidad y calidad de las estrategias que el alumnado pone en juego cuando aprende son una de las causas que diferencian a los buenos estudiantes de los que no lo son tanto. En Educación Superior, se espera que el alumnado disponga de intereses intrínsecos por aprender, se comprometa en la utilización de estrategias de procesamiento profundo, se esfuerce, asuma responsabilidades en las tareas y disponga de estrategias de autorregulación (Garrello y Rinaudo, 2012).

Diferentes investigaciones revelan cómo las estrategias de aprendizaje de estudiantes universitarios se relacionan con sus resultados académicos (De la Fuente et al., 2008; Garavalia y Gredler, 2002; Pintrinch y Schunk, 2004), así como con su desarrollo vital y profesional (Jiménez et al., 2018). Dicha vinculación está asociada tanto a variables de tipo individual como la edad, género, estilo de aprendizaje, motivación, autorregulación y metas de aprendizaje (Bruinsma, 2004; Cano, 2000; Martín y Camarero, 2001; Valle et al., 2006; Wolters, 2004) así como variables contextuales, referidas a la organización del plan de estudios universitario, estilos de enseñanza del profesorado y métodos instruccionales empleados (Cope y Staehr, 2005; Jansen, 2004).

Además, el uso de metodologías innovadoras centradas en el aprendizaje, que aúnan la metodología expositiva con elementos interactivos, trabajo autónomo y cooperativo, resolución de problemas, prácticas de laboratorio, tutorías en el aula, evaluación formativa etc., mejoran tanto las estrategias como los enfoques de aprendizaje del estudiante, al tiempo que obtienen mejores calificaciones (Gargallo, Morera y García, 2015). En este sentido, autores como Backman y Larsson (2014) indican cómo una de las mejores maneras para fomentar esa autonomía en el aula es mediante el diseño y puesta en práctica de sesiones en las que el estudiante pueda ser parte activa del proceso de enseñanza y utilice el aprendizaje para plantear sus sesiones a los compañeros (Hortigüela et al., 2015).

Estas expectativas no siempre son realistas, pues existen estudiantes que tienden a adoptar un nivel superficial en la lectura de materiales, cumpliendo mínimamente con las demandas del curso y utilizando sólo estrategias de memorización (Boekaerts y Martens, 2006). Incluso, distintas investigaciones (Herrero, González y Marín, 2015; Vallejo y Molina, 2011) revelan cómo el estudiante considera que la clase magistral sigue siendo una de las metodologías con mayor presencia en el aula universitaria, tras el aprendizaje cooperativo y el trabajo autónomo del alumno, a pesar de ser éstas más enriquecedoras.

Centrados en la formación del futuro docente, García, Castañeda y Mansilla (2018) subrayan cómo un aprendiz docente debe incorporar habilidades y competencias que le capaciten para ser un agente educativo activo, creativo, innovador y reflexivo; para ello, su intervención educativa debe implementar procesos de aprendizaje autorregulado a 
partir de herramientas con las que el estudiante se conozca mejor, planifique y ponga en práctica determinadas estrategias según la tarea a desarrollar y realice de manera adecuada la toma de decisiones para el desarrollo de un aprendizaje a lo largo de la vida. En este sentido, Guzmán (2018) sostiene cómo la formación del maestro debe tener en cuenta las necesidades, intereses y nivel de conocimientos del estudiante, empleando diferentes estrategias de aprendizaje capaces de propiciar la aplicación de lo aprendido y utilizar la evaluación en sentido formativo.

Otros estudios vinculados al área de Ciencias de la Salud demuestran cómo los estudiantes de Enfermería de la Universidad de Pamplona realizan con frecuencia actividades propias del aprendizaje autónomo (Uribe, 2012); de este modo, se establece un plan formativo que interviene en aspectos determinantes para el buen rendimiento académico del estudiante en Educación Superior como pueda ser la motivación, planificación eficaz de actividades, disposición para adquirir y mantener el conocimiento, la toma de decisiones, comunicación, autoconcepto, poder, esperanza y desempeño eficaz del rol del estudiante.

No obstante, se encuentra poca fundamentación teórica que justifique las diferencias de edad sobre los procesos de estudio en alumnado de Educación Superior. Algunas investigaciones (Hernández-Pina et al., 2002; Richardson, 1995; Sadler-Smith, 1996) han demostrado cómo el alumnado de mayor edad tiende a utilizar un enfoque más profundo, mientras que los estudiantes de menor edad adoptan un enfoque más superficial; por su parte, Zhang (2000) expone cómo el alumnado de mayor edad adopta el enfoque de logro en menor medida.

No obstante, el mero hecho de que el alumnado de Educación Superior alcance una determinada edad no significa que disponga de estrategias de aprendizaje maduras, siendo necesario el entrenamiento hacia la incorporación de aprendizajes adecuados a las materias que se estudian en las distintas titulaciones (Carbonero y Coromoto, 2006). En este sentido, Rivero y Loyola (2016) exponen cómo las estrategias de aprendizaje autónomo en estudiantes universitarios adscritos a la carrera de Derecho están igualmente desarrolladas independientemente de la cantidad de años que el estudiante haya cursado su carrera.

Por ello, el objetivo de esta investigación es explorar las estrategias de trabajo autónomo de los estudiantes noveles universitarios de Educación. De este modo, se realiza un análisis preliminar de las destrezas y habilidades hacia el aprendizaje autorregulado de que dispone el estudiante una vez accede al sistema universitario, ajustándose a sus necesidades e intereses, para así poder mejorar la calidad del proceso de enseñanzaaprendizaje. No obstante, y de modo más concreto, este trabajo pretende desarrollar los siguientes objetivos específicos:

- Analizar las destrezas para la búsqueda y elaboración de materiales y actividades complementarias de ampliación del material propuesto por el profesor.

- Estudiar las estrategias relacionadas con la implicación del alumno en tareas grupales y de relación con otros compañeros.

- Examinar las destrezas relacionadas con el trabajo intelectual sobre el contenido. 
- Explorar las habilidades del estudiante universitario para la planificación de los tiempos y la programación de las tareas de estudio, elaboración de trabajos y evaluación de los procedimientos de aprendizaje.

- Examinar las estrategias vinculadas con el estudio para los exámenes.

- Conocer el nivel de participación del estudiante en el proceso de enseñanzaaprendizaje.

- Comprobar si la edad del estudiante universitario determina el desarrollo de estrategias de aprendizaje autónomo en su proceso de enseñanza-aprendizaje.

\section{Método}

Para dar respuesta a los objetivos planteados, se lleva a cabo un estudio de carácter cuantitativo basado en una metodología de investigación no experimental de tipo descriptivo-correlacional, utilizándose la encuesta como principal técnica para la recogida de datos.

\section{Variables}

Las variables de esta investigación se corresponden con las distintas dimensiones planteadas en el instrumento de recogida de datos para la valoración de las estrategias de trabajo autónomo del estudiante. Estos tienen en consideración los objetivos específicos planteados, utilizándose las siguientes nomenclaturas para facilitar su lectura:

- Estrategias de ampliación (AMP).

- Estrategias de colaboración (COL).

- Estrategias de conceptualización (CON).

- Estrategias de planificación (PLA).

- Estrategias de preparación de exámenes (PRE).

- Estrategias de participación (PAR).

Además, todas estas variables incluyen diversos ítems descritos posteriormente en el instrumento de recogida de datos, permitiendo obtener unos resultados significativos para cada una de ellas. Incluso, se han tenido en cuenta variables sociodemográficas tales como: género, edad, modalidad de acceso a la Educación Superior y Grado en Educación en que se encuentran matriculados.

\section{Muestra}

La muestra objeto de estudio de esta investigación está integrada por 407 estudiantes matriculados oficialmente durante el curso 2017/18 en el primer curso de los Grados en Educación Infantil, Educación Primaria y Educación Social de la Universidad de Jaén (n $=614)$. Se pretende analizar esta área de interés para, de este modo, comprender el planteamiento del proceso de aprendizaje del estudiante universitario, adaptándolo a las necesidades y características del estudiante.

Para la selección de la muestra se ha utilizado un muestreo no probabilístico de tipo casual o incidental, participando todos los estudiantes de primer curso de los Grados en Educación que cumplimentaron el cuestionario. Para el cálculo de la muestra participante se ha utilizado la fórmula para poblaciones con menos de 100.000 sujetos, 
con un nivel de confianza del $95 \%$ y un error de estimación máximo de 4\%. Además, se han tenido en cuenta como requisitos para la selección de la muestra las orientaciones propuestas por Hernández, Fernández y Baptista (2014) relacionadas con: el acceso a la muestra y su aceptación, la necesaria atención a las imposiciones del estudio, así como la disponibilidad de los participantes para el proceso de recogida de datos de manera óptima y eficaz.

Así pues, un $87,4 \%$ de la muestra son mujeres, frente al 12,6\% que está compuesto por hombres. Se trata, en un 50,9\% de los casos, de estudiantes menores de 20 años, frente al $30,0 \%$ cuya edad se sitúa entre 21 y 30 años y un 6,5\% mayores de 31 años. Al valorar la modalidad de acceso, un $63,5 \%$ ha accedido a dichos Grados tras cursar Bachillerato de Humanidades y Ciencias Sociales, un $21,6 \%$ ha cursado anteriormente un Ciclo Formativo de Grado Superior (Integración Social, Educación Infantil, etc.), un 10,0\% Bachillerato de Ciencias y Tecnología, y finalmente, un 4,9\% a través de las pruebas de acceso para mayores de 25 años. En cuanto al Grado en que se encuentran matriculados, un 39,0\% pertenece al Grado en Educación Primaria, un 33,6\% al Grado en Educación Infantil y un 27.4\% al Grado en Educación Social de la Universidad de Jaén.

\section{Instrumento de recogida de datos}

Para la recogida de datos, se ha utilizado la técnica de la encuesta a través del "Cuestionario de Estrategias de Trabajo Autónomo, CETA" (López, 2010). Se trata de un instrumento centrado en la medida de las estrategias de trabajo autónomo en estudiantes universitarios, en el contexto de la forma actual de la enseñanza superior, siendo éste uno de los pilares para la formación en competencias.

Dicho instrumento consiste en una escala tipo Likert compuesta por 45 ítems, cuyas opciones de respuesta oscilan entre 1 y 5 (siendo $1=$ nunca; $2=$ pocas veces; $3=$ algunas veces; $4=$ muchas veces y $5=$ siempre) y dividida en 6 sub-escalas tales como:

- Estrategias de ampliación: relacionadas con la búsqueda y elaboración de materiales y actividades complementarias de ampliación del material (9 ítems).

- Estrategias de colaboración: relacionadas con la implicación del alumno en tareas grupales y de relación con otros compañeros (11 ítems).

- Estrategias de conceptualización: vinculadas con el trabajo intelectual sobre el contenido (8 ítems).

- Estrategias de planificación: recoge aspectos relativos a la planificación de los tiempos y programación de las tareas, tanto de estudio como de elaboración de trabajos y evaluación de los procedimientos de aprendizaje ( 5 ítems).

- Estrategias de preparación de exámenes: especialmente selección de puntos importantes sobre el temario y la realización de actividades de repaso (6 ítems).

- Estrategias de participación: describen el nivel de implicación del alumno en el proceso de enseñanza-aprendizaje (asistencia a clase, aclaración de dudas, participación en el aula, tutorías, etc.) (6 ítems).

Dicho instrumento posee unas adecuadas cualidades psicométricas, habiéndose comprobado, para el análisis del poder discriminativo de los ítems, su adecuada distribución, así como el elevado coeficiente de fiabilidad (0,898). Por su parte, el índice Kaisar-Meyer-Olkin (KMO $=0,845$ ) informa de una correcta adecuación muestral, 
existiendo interrelaciones significativas entre las variables que alertan de la adecuación de los datos para el modelo de análisis factorial.

\section{Análisis de datos}

Para el tratamiento estadístico de los datos se ha utilizado el programa SPSS (versión 21 para Windows) al considerarlo un recurso idóneo para dicho trabajo. De este modo, se ha llevado a cabo un análisis descriptivo de las variables, así como de los ítems que componen cada una de ellas. Para ello, se ha utilizado estadísticos como la Media (M), Desviación Típica (DT), Coeficiente de Asimetría de Pearson (CAP) y el Coeficiente de Apuntamiento de Fisher (CAF).

A continuación, el análisis de varianza (ANOVA) y la prueba Tukey realizada a posteriori ha permitido comprobar la existencia de diferencias estadísticamente significativas entre las variables del cuestionario y la "edad" del estudiante universitario. En este caso, se ha utilizado un valor de $\mathrm{p}<0,05$ como diferencia estadísticamente significativa, determinado por un nivel de confianza del $95 \%$ y un margen de error del $5 \%$.

\section{Resultados}

El análisis general de las variables para este estudio revela unos valores medios similares para todas las estrategias, lo que indica que todas están medianamente desarrolladas. Sin embargo, las estrategias más desarrolladas por los estudiantes universitarios noveles adscritos a los Grados en Educación son la preparación de exámenes, centradas en la selección de puntos importantes y actividades de repaso y las estrategias de conceptualización, vinculadas al trabajo intelectual sobre el contenido de las asignaturas. Por el contrario, las menos desarrolladas se relacionan con el desarrollo de habilidades y estrategias de colaboración y relación con otros compañeros y las estrategias de ampliación del material de las asignaturas propuestos por el profesor.

Cuadro 1. Análisis descriptivo para las variables del "Cuestionario CETA"

\begin{tabular}{lccccccc}
\hline \multicolumn{1}{c}{ VARIABLES } & N & MIN & MAX & M & SD & CA $_{\mathbf{p}}$ & CA $^{\mathbf{F}}$ \\
\hline Preparación de exámenes (PRE) & 407 & 1 & 5 & 3,98 & 0,678 & 0,457 & 0,135 \\
Conceptualización (CON) & 405 & 1 & 5 & 3,76 & 0,703 & $-0,323$ & $-0,133$ \\
Participación (PAR) & 407 & 1 & 5 & 3,35 & 0,745 & 0,426 & 1,402 \\
Planificación (PLA) & 407 & 1 & 5 & 3,02 & 0,769 & $-0,014$ & $-0,291$ \\
Colaboración (COL) & 403 & 1 & 5 & 3,00 & 0,744 & 0,271 & 0,622 \\
Ampliación (AMP) & 406 & 1 & 5 & 2,68 & 0,759 & 0,136 & 0,343 \\
\hline
\end{tabular}

Nota: $\mathrm{CA}_{\mathrm{P}}$ : Coeficiencia de asimetría de Pearson. $\mathrm{CA}^{\mathrm{F}}$ : Coeficiente de apuntamiento de Fisher.

Fuente: Elaboración propia.

Centrados en el análisis para las estrategias de ampliación, los resultados demuestran cómo los estudiantes preparan sus exámenes teniendo en cuenta todo el material de la asignatura y no sólo los apuntes de clase. Además, afirman realizar búsquedas en libros o en Internet para aclarar dudas o ampliar conceptos. De este modo, consideran Internet como el mejor recurso para la búsqueda de información a través de la elaboración de bases de datos y la consulta de fuentes de información adecuadas. No obstante, completan su estudio con la realización de actividades complementarias y lecturas, mostrándose más remisos para la consulta de bibliografía recomendada. 
Cuadro 2. Análisis descriptivo para la variable "Estrategias de ampliación”

\begin{tabular}{lcc}
\hline & M & SD \\
\hline $\begin{array}{l}\text { 25. Preparo los exámenes teniendo en cuenta todo el material, no sólo los } \\
\text { apuntes }\end{array}$ & 4,06 & 0,980 \\
$\begin{array}{l}\text { 45. Cuando me surgen dudas, o para ampliar algún concepto, realizo } \\
\text { búsquedas en libros o en Internet }\end{array}$ & 3,28 & 1,273 \\
18. Busco datos, relativos al tema, en Internet & 2,82 & 1,189 \\
8. Busco más información navegando por Internet & 2,80 & 1,237 \\
9. Realizo actividades complementarias & 2,73 & 1,159 \\
40. Consulto otros materiales bibliográficos o páginas de Internet que ayuden & 2,67 & 1,223 \\
o mejoren la comprensión & & \\
16. Elaboro una base de datos con toda la información obtenida en el & 2,63 & 1,055 \\
$\quad$ desarrollo del trabajo & 2,62 & 1,103 \\
14. Completo el estudio con lecturas/trabajos complementarios & 2,32 & 1,186 \\
19. Consulto bibliografía recomendada &
\end{tabular}

Fuente: Elaboración propia.

Para las estrategias de colaboración, los estudiantes noveles encuestados afirman consultar dudas con los compañeros de clase y revisar sus apuntes de clase. Incluso, sostienen intercambiar documentos y direcciones Webs de utilidad para la resolución de actividades de clase. Definen el trabajo colaborativo como una herramienta para resolver un problema o investigar sobre algún tema de interés, integrando las aportaciones de otros compañeros en los resúmenes de los temas.

Los estudiantes conocen y utilizan los recursos que proporciona el campus universitario, intercambiando los resúmenes de los temas con otros compañeros. No obstante, se muestran más reacios a repartir entre los compañeros la tarea de elaborar sinopsis de los libros incluidos en la bibliografía básica, así como en la búsqueda y préstamo de libros y manuales de la biblioteca de la Universidad de Jaén.

Cuadro 3. Análisis descriptivo para la variable "Estrategias de colaboración"

\begin{tabular}{lcc}
\hline & M & SD \\
\hline $\begin{array}{l}\text { 27. Consulto con los compañeros las dudas que se me plantean en el estudio del } \\
\text { tema }\end{array}$ & 4,05 & 0,973 \\
$\begin{array}{l}\text { 37. Trabajo en colaboración para resolver un problema o investigar algo } \\
\text { 38. Reviso los apuntes de los compañeros para ver si aclaran las dudas }\end{array}$ & 3,58 & 1,034 \\
$\begin{array}{l}\text { 26. Intercambio con compañeros documentos, direcciones Webs, que nos pueden } \\
\text { ser útiles para el desarrollo de las actividades }\end{array}$ & 3,39 & 1,201 \\
$\begin{array}{l}\text { 21. En la elaboración de resúmenes de cada uno de los temas, integro las } \\
\text { aportaciones hechas por otros compañeros en clase }\end{array}$ & 1,264 \\
$\begin{array}{l}\text { 15. Conozco y utilizo los recursos que proporciona el campus } \\
\begin{array}{l}\text { 33. Pongo a disposición de los compañeros los apuntes que he elaborado para } \\
\text { facilitar el estudio del temario }\end{array}\end{array}$ & 3,0508 \\
$\begin{array}{l}\text { 24. Cuando descubro aportaciones nuevas en documentos comentarios a la } \\
\text { bibliografía recomendada, lo comparto con los compañeros }\end{array}$ & 2,053 \\
$\begin{array}{l}\text { 22. Intercambio los resúmenes de los temas con otros compañeros } \\
\text { 31. Reparto con algunos compañeros los libros de la bibliografía básica, }\end{array} \quad 2,77$ & 1,248 \\
$\quad$ elaborando sinopsis de cada uno de ellos para compartirlos & 2,72 & 1,360 \\
23. Me organizo con los compañeros para pedir libros a la biblioteca & 2,39 & 2,003 \\
\hline
\end{tabular}

Fuente: Elaboración propia. 
Centrados en el desarrollo de las estrategias de conceptualización, el estudiante manifiesta utilizar diferentes recursos tales como resúmenes, esquemas o cuadros sinópticos, mapas y esquemas globales para trabajar los contenidos de cada tema, así como mapas conceptuales con las nociones más importantes de cada apartado. Además, manifiesta recopilar los contenidos más significativos como notas de estudio, leer y esquematizar los temas, haciendo uso de notas al margen para sintetizar lo leído y aportar una síntesis personal del contenido.

Cuadro 4. Análisis descriptivo para la variable "Estrategias de conceptualización"

\begin{tabular}{lcc}
\hline & M & SD \\
\hline 32. Confecciono un resumen de cada tema & 4,13 & 1,121 \\
$\begin{array}{l}\text { 3. Estudio con esquemas, resúmenes y cuadros sinópticos de los contenidos de } \\
\text { cada tema }\end{array}$ & 4,13 & 1,026 \\
$\begin{array}{l}\text { 43. Recopilo los contenidos que considero más importantes a modo de notas de } \\
\text { estudio }\end{array}$ & 3,76 & 1,046 \\
$\begin{array}{l}\text { 13. Leo y esquematizo los contenidos } \\
\text { 5. Cuando inicio la lectura de un tema, escribo notas que posteriormente me } \\
\text { sirven de síntesis de lo leído }\end{array}$ & 3,73 & 0,988 \\
7. Realizo mapas conceptuales y esquemas globales & 1,243 \\
6. Construyo una síntesis personal de los contenidos & 3,46 & 1,201 \\
11. Realizo un mapa conceptual de los conceptos más importantes de cada & 3,32 & 1,171 \\
apartado & 3,19 & 1,230 \\
\hline
\end{tabular}
Fuente: Elaboración propia.

En cuanto a las habilidades y estrategias de planificación, el alumnado afirma repartirse el tiempo para estudiar los contenidos y elaborar los trabajos, planificando el tiempo disponible para cada asignatura y trabajo práctico, así como las estrategias de estudio a desarrollar. Además, el estudiante evalúa el proceso de aprendizaje final, mostrándose menos convencido de elaborar un plan de trabajo al inicio del cuatrimestre con el tiempo dedicado a cada asignatura y la fecha de los exámenes.

Cuadro 5. Análisis descriptivo para la variable "Estrategias de planificación"

\begin{tabular}{lcc}
\hline & M & SD \\
\hline $\begin{array}{lc}\text { 30. Reparto el tiempo para el estudio de contenidos y la elaboración de los trabajos } \\
\text { de cada tema }\end{array}$ & 3,63 & 0,985 \\
44. Planifico el tiempo de que dispongo de cada asignatura y trabajo práctico & 3,53 & 1,157 \\
12. Planifico los tiempos y las estrategias de estudio & 3,31 & 1,115 \\
17. Evalúa el proceso de aprendizaje final & 2,82 & 1,212 \\
10. Al empezar el cuatrimestre, hago por escrito un plan de trabajo, reflejando el & 2,34 & 1,186 \\
\hline
\end{tabular}
Fuente: Elaboración propia.

Respecto al desarrollo de estrategias para la preparación de exámenes, el alumnado encuestado afirma repasar las indicaciones aportadas por el profesor al inicio del curso Antes del examen, dedica unos días al repaso de los contenidos para aclarar las dudas finales, estando centrado en lo que el profesor marca como importante. Así pues, y para el estudio de los temas, realiza una primera lectura rápida y después, otra más detenida en la que recoge la información más relevante, llevando a cabo una selección de los puntos más importantes. Además, en los debates de clase tiene en cuenta las aportaciones de los compañeros para realizar la suya propia. 
Cuadro 6. Análisis descriptivo para la variable "Estrategias de preparación de exámenes"

\begin{tabular}{lcc}
\hline & M & SD \\
\hline $\begin{array}{l}\text { 36. Repaso las indicaciones que el profesor nos ha dado a lo largo del curso } \\
\text { 34. Antes de los exámenes, dedico unos días de repaso para aclarar dudas finales }\end{array}$ & 4,10 & 0,843 \\
$\begin{array}{l}\text { 35. Para preparar el examen, me baso principalmente en los aspectos que el } \\
\text { profesor marca como importantes }\end{array}$ & 3,051 \\
$\begin{array}{l}\text { 42. Realizo una primera lectura rápida y después otra más detenida con copia o } \\
\text { trascripción de lo más relevante }\end{array}$ & 3,078 \\
$\begin{array}{l}\text { 29. Cuando hay debate, tengo en cuenta las aportaciones con los compañeros para } \\
\text { realizar la mía }\end{array}$ & 3,62 & 1,033 \\
$\begin{array}{l}\text { 20. Leo todo el material de la asignatura y hago una selección de los puntos más } \\
\text { importantes para trabajarlos }\end{array}$ & 3,41 & 1,094 \\
\hline
\end{tabular}

Fuente: Elaboración propia.

Finalmente, y en cuenta a las estrategias de participación, el alumnado encuestado manifiesta corregir las actividades planteadas para comprobar los conocimientos de que dispone tomando notas de las respuestas del profesor ante las dudas planteadas, así como también manifiesta anotarlas para examinarlas en profundidad tras una segunda lectura o consultarlas al profesor en clase y/o tutorías. Además, el alumnado afirma seguir, aprovechar y participar en las clases, respondiendo a las preguntas planteadas.

Cuadro 7. Análisis descriptivo para la variable "Estrategias de participación"

\begin{tabular}{lcc}
\hline & M & SD \\
\hline 30. Corrijo las actividades propuestas para comprobar mis conocimientos & 4,03 & 0,932 \\
1.Tomo nota de las respuestas del profesor a las dudas propias o de los & 3,65 & 1,011 \\
$\quad$ compañeros & 3,49 & 1,059 \\
41. Sigo, aprovecho y participo en las clases & 3,27 & 1,084 \\
2. Anoto mis dudas para consultarlas más a fondo en una segunda lectura & 3,26 & 1,068 \\
28. Respondo a las preguntas planteadas en clase & 3,10 & 1,907 \\
4. Aclaro las dudas con el profesor en clase o en tutorías & &
\end{tabular}

Fuente: Elaboración propia.

El análisis de varianza realizado (ANOVA) entre las variables del cuestionario según la "edad del estudiante" ha permitido detectar diferencias estadísticamente significativas para las variables vinculadas con el desarrollo de estrategias de preparación de los exámenes $(\mathrm{F}(4,190)=2,629, \mathrm{p}=0,035)$, trabajo intelectual sobre el contenido $(\mathrm{F}(4,190)$ $=3,384, \mathrm{p}=0,011)$ y participación en el proceso de enseñanza-aprendizaje $(\mathrm{F}(4,190)=$ $3,842, \mathrm{p}=0,005)$. También se muestran diferencias a nivel estadístico para el desarrollo de estrategias de colaboración en tareas grupales y relación con su grupo de iguales $(\mathrm{F}(4,190)=2,422, \mathrm{p}=0,050)$ y para la búsqueda $\mathrm{y}$ elaboración de material complementario de estudio $(\mathrm{F}(4,190)=3,600, \mathrm{p}=0,007)$. No obstante, no se muestran diferencias en las valoraciones del estudiante hacia el desarrollo de estrategias de planificación del tiempo, de tareas y evaluación del procedimiento de aprendizaje según su edad $(\mathrm{F}(4,190)=1,395, \mathrm{p}=0,237)$.

La prueba de Tukey realizada a posteriori revela cómo dichas diferencias se sitúan entre el alumnado más joven que acaba de comenzar sus estudios universitarios, siendo éstos menores de 20 años y aquel otro de mayor edad (de más de 31 años). En este caso, y tal y como se muestra en la tabla adjunta, las valoraciones resultan más favorables para el alumnado universitario más joven, mostrando mayor disponibilidad hacia el desarrollo 
de determinadas estrategias basadas en el aprendizaje autónomo en el proceso de enseñanza-aprendizaje en Educación Superior.

Cuadro 8. ANOVA entre las variables del cuestionario “CETA” según la edad

\begin{tabular}{lcccccc}
\hline \multirow{2}{*}{ VARIABLE } & \multicolumn{4}{c}{ M } & \multirow{2}{*}{ SiG. } & TUKEY \\
\cline { 2 - 5 } & $<$ 20 años & $\mathbf{2 1 - 2 9}$ años & $>$ 30 años & & \\
\hline Preparación de exámenes & $3,69^{*}$ & 3,92 & $4,27^{*}$ & 0,036 & $<20->30$ años \\
Conceptualización & $3,49^{*}$ & 3,75 & $3,99^{*}$ & 0,011 & $<20->30$ años \\
Participación & $3,37^{*}$ & 3,61 & $4,35^{*}$ & 0,005 & $<20->30$ años \\
Planificación & 3,05 & 3,35 & 3,10 & 0,237 & - \\
Colaboración & $2,93^{*}$ & 3,15 & $3,70^{*}$ & 0,050 & $<20->30$ años \\
Ampliación & $2,82^{*}$ & 3,10 & $3,65^{*}$ & 0,007 & $<20->30$ años \\
\hline Nota: *Relación significativa & a nivel estadístico entre & variables; & - No existen relaciones \\
significativas a nivel estadístico entre variables. & & & & \\
Fuente: Elaboración propia. & & & & &
\end{tabular}

\section{Discusión y conclusiones}

Este trabajo ha permitido realizar un análisis preliminar acerca de las estrategias de trabajo autónomo en estudiantes universitarios noveles de Educación. Sin duda, se trata de una investigación de enorme interés, pues acerca al profesorado universitario hacia el conocimiento de las estrategias y habilidades de que dispone el estudiante universitario para, de este modo, adaptar el proceso de enseñanza-aprendizaje a dichas circunstancias. Además, permite al estudiante universitario adecuar sus preferencias de aprendizaje al perfil académico de las titulaciones de Educación, así como también supone un factor que propicia la mejora de las acciones formativas para los estudiantes de nuevo ingreso.

Así pues, las estrategias más desarrolladas por los estudiantes se relacionan con la preparación de exámenes y el trabajo intelectual del estudiante sobre el contenido de las asignaturas incluidas en los planes de estudio. Ello evidencia la aceptación y el compromiso del estudiante universitario hacia el nuevo paradigma educativo diseñado en la actualidad en la Educación Superior. Se trata de un cambio que potencia la construcción personal del aprendizaje por parte del estudiante, a partir de su participación activa, autónoma y autorregulada para la elaboración de los contenidos. Ello implica la puesta en práctica de estrategias de aprendizaje para la evaluación, de modo que el estudiante no se limite a añadir de manera mecánica los conocimientos en su memoria, sino que debe realizar una serie de actividades para facilitar su comprensión y asimilación en la estructura cognitiva de que dispone.

No obstante, también se aprecia en el estudiante el desarrollo de estrategias relacionadas con su participación en el proceso de enseñanza-aprendizaje, la planificación temporal, realización de tareas y evaluación del aprendizaje, así como su colaboración en tareas grupales y de relación con otros compañeros. Por tanto, la labor del profesor universitario ante este nuevo escenario no se limita a transmitir información, sino en potenciar la actividad del estudiante para filtrar la información, organizarla, procesarla, construir los contenidos de aprendizaje y desarrollar las habilidades y competencias de aprendizaje.

Investigaciones como las desarrolladas por Herrero, González y Marín (2015) así como Vallejo y Molina (2011) exponen la importancia otorgada por el estudiante hacia el trabajo autónomo, a pesar de ser la clase magistral la que mayor presencia tiene en el 
aula de Educación Superior. El hecho de aunar procedimientos de enseñanza basados en metodologías expositivas que tengan en cuenta el trabajo autónomo y cooperativo del estudiante mejora las estrategias de aprendizaje, así como los resultados académicos del alumnado universitario (Gargallo, Morera y García, 2015). En cuanto a la estrategia de aprendizaje basada en la colaboración, debe destacarse la actitud positiva del estudiante hacia este tipo de tareas cooperativas, entendiéndolas como oportunidades de aprendizaje que persiguen la mejora y optimización de su rendimiento académico.

Finalmente, se destaca la estrategia de ampliación como la menos desarrollada por los estudiantes participantes en la investigación, estando vinculada a la búsqueda y elaboración de materiales y actividades para el desarrollo de la asignatura. Estos datos concuerdan con los aportados por Boekaerts y Martens (2006) quiénes aluden a la puesta en práctica de estrategias de memorización en el estudiante desde una pedagogía de mínimos, lo que le hace cumplir con los criterios de evaluación básicos propuestos en las distintas asignaturas. Este resultado puede deberse a la falta de motivación del estudiante para el desarrollo de sus estudios universitarios; en general, se muestran poco preocupados por el aprendizaje, centrando su interés tan sólo en lograr un aprobado y obtener una titulación universitaria. Ante esta situación, la institución universitaria y, más concretamente, el profesorado debería plantearse los motivos que justifican la falta de interés del estudiante de Educación hacia sus estudios, pues en un futuro próximo serán los responsables de la educación de los más pequeños de nuestra sociedad.

Además, se ha demostrado cómo los estudiantes más jóvenes desarrollan mayores estrategias de aprendizaje autónomo que aquellos otros que poseen mayor edad. Estos resultados guardan relación con las conclusiones expuestas por Zhang (2000), quien demuestra cómo el estudiante de menor edad posee un mayor enfoque de logro hacia el aprendizaje. En cambio, otros autores (Hernández-Pina et al., 2002; Richardson, 1995; Sadler-Smith, 1996) señalan al alumnado más joven como aquel que tiende a adoptar un enfoque de aprendizaje más superficial.

Dichas conclusiones pueden justificarse por el hecho de que el estudiante universitario de mayor edad cuenta con mayor nivel de responsabilidades personales y/o profesionales que les llevan a desarrollar dichos estudios universitarios de manera complementaria. En este sentido, y dado el nuevo paradigma en Educación Superior, no llegan a realizar una adecuada inmersión y adecuación de su rol a las exigencias del sistema universitario, lo que perjudica en su rendimiento académico. Por el contrario, el estudiante más joven dispone de una formación reciente durante sus estudios de Educación Secundaria Obligatoria que le lleva a la puesta en práctica de dichas estrategias que potencian un aprendizaje autorregulado.

En definitiva, y tal y como apunta Martínez (2015), para el desarrollo de una Educación Superior de calidad, la enseñanza no debe sólo centrarse en el ámbito profesional, sino que debe abarcar una formación integral del estudiante, haciendo hincapié en la adquisición de valores, capacidades y habilidades. En este contexto, el aprendizaje autorregulado supone una metodología innovadora, capaz de facilitar un aprendizaje más reflexivo, responsable, autónomo y cooperativo (Biggs, 2010; Domingo y Gómez, 2014).

No obstante, este trabajo presenta algunas limitaciones, pues se ha utilizado como instrumento de recogida de datos un cuestionario, lo cual puede generar problemas de deseabilidad social y sinceridad entre los encuestados. Asimismo, el hecho de acceder a 
estudiantes noveles del área de Educación de la Universidad de Jaén supone un estudio de caso, generando dificultades para la generalización de los datos a otras muestras.

Por ello, y para futuras investigaciones, se pretenden crear grupos de discusión que aporten datos cualitativos al estudio sobre las estrategias de aprendizaje autónomo en estudiantes universitarios. Además, resulta necesario ampliar la muestra objeto de estudio para estudiantes de otros Grados y ámbitos de conocimiento, incluso comparar el desarrollo de tales estrategias entre universitarios matriculados en distintas universidades (públicas y privadas).

\section{Referencias}

Aguado, D., González, A., Antúnez, M. y De Dios, T. (2017). Evaluación de competencias transversales en universitarios. Propiedades psicométricas iniciales del Cuestionario de Competencias Transversales. REICE. Revista Iberoamericana sobre Calidad, Eficacia y Cambio en Educación, 15(2), 129-152. https://doi.org/10.15366/reice2017.15.2.007

Backman, E. y Larsson, H. (2014). What should a physical education teacher know? An analysis of learning outcomes for future physical education teachers in Sweden. Physical Eduction and Sport Pedagogy, 3(12), 27-31. https://doi.org/10.1080/17408989.2014.946007.

Bahamón, M., Vianchá, M., Alarcón, L. y Bohórquez, C. (2013). Estilos y estrategias de aprendizaje relacionados con el logro académico en estudiantes universitarios. Pensamiento Psicológico, 11(1), 115-129.

Biggs, J. (2010). Calidad del aprendizaje universitario. Madrid: Narcea.

Boekaerts, M. y Martens, R. (2006). Motivated learning: What it is and how can it be enhanced? En L. Verschaffel, F. Dochy, M. Boekaert y S. Vosniadou (Eds.), Instructional psychology: past, present and future trends. Sixteen essays in honour of Erik De Corte (pp. 113-130). Oxford: Elsevier.

Bruinsma, M. (2004). Motivation, cognitive processing and achievement in higher education. Learning and Instruction, 14, 549-568. https://doi.org/10.1016/j.learninstruc.2004.09.001

Cano, F. (2000). Diferencias de género en estrategias y estilos de aprendizaje. Psicothema, 12(3), 362-367.

Carbonero, M. A. y Coromoto, J. (2006). Entrenamiento de alumnos de Educación Superior en estrategias de aprendizaje en matemáticas. Psicothema, 18(3), 348-352.

Coll, C., Mauri, T. y Rochera, M. J. (2012). La práctica de evaluación como contexto para aprender a ser aprendiz competente. Profesorado. Revista de Curriculum y Formación del Profesorado, 16(1), 50-57.

Cope, C. y Staehr, L. (2005). Improving student`s learning approaches through intervention in an information systems learning environment. Studies in Higuer Education, 30, 181-197. https://doi.org/10.1080/03075070500043275

Cuadrado, I., Monroy, F. A. y Montaño, A. (2011). Características propias de los estilos de aprendizaje de los estudiantes de maestros de educación infantil. International Journal of Developmental and Educational Psychology, 1(3), 217-226.

De la Fuente, J., Pichardo, M. C., Justicia, F. y García-Berbén, A. B. (2008). Enfoques de aprendizaje, autorregulación y rendimiento en tres universidades europeas. Psicothema, $20(4), 705-711$. 
Domingo, A. y Gómez, V. (2014). La práctica reflexiva. Bases, modelos e instrumentos. Madrid: Narcea.

Fernández-Molina, M., González, V. y Del Molino, G. (2011). Perfil del alumnado universitario de educación infantil. Un estudio descriptivo desde los inicios del espacio europeo de educación superior hasta los estudios de grado. Revista de Investigación Educativa, 29(1), 187-203.

Freiberg, A., Ledesma, R. y Fernández, M. (2017). Estilos y estrategias de aprendizaje en estudiantes universitarios de Buenos Aires. Revista de Psicología, 35(2), 535-573. https://doi.org/10.18800/psico.201702.006

Garavalia, L. S. y Gredler, M. E. (2002). Prior achievement aptitude and use of learning strategies as predictors of college Student achievement. College Student Journal, 36, 616626.

García, C., Castañeda, E. y Mansilla, J. M. (2018). Experiencia de innovación en el aula desde la autorregulación y los estilos de aprendizaje. Tendencias Pedagógicas, 31, 137-148. https://doi.org/10.15366/tp2018.31.008

García-Valcárcel, A. y Tejedor, F. J. (2017). Percepción de los estudiantes sobre el valor de las TIC en sus estrategias de aprendizaje y su relación con el rendimiento. Educación XX1, 2O(2), 137-159. https://doi.org/10.5944/educXX1.13447

Gargallo, B., Morera, I. y García, E. (2015). Metodología innovadora en la universidad. Sus efectos sobre los procesos de aprendizaje de los estudiantes universitarios. Anales de Psicología, 31(3), 901-915.

Gargallo, B., Suárez, J. M. y Pérez, C. (2009). El cuestionario CEVEAPEU. Un instrumento para la evaluación de las estrategias de aprendizaje de los estudiantes universitarios. RELIEVE, 15(2), 1-31. https://doi.org/10.7203/relieve.15.2.4156

Garrello, M. V. y Rianudo, M. C. (2012). Rasgos del contexto para la promoción del desarrollo académico y la creatividad. Estudio de diseño con estudiantes universitarios. REICE. Revista Iberoamericana sobre Calidad, Eficacia y Cambio en Educación, 1O(2), 159-179.

Garrote, D., Garrote, C. y Jiménez, S. (2016). Factores influyentes en motivación y estrategias de aprendizaje en los alumnos de Grado. REICE. Revista Iberoamericana sobre Calidad, Eficacia y Cambio en Educación, 14(2), 31-44. https://doi.org/10.15366/reice2016.14.2.002

Guzmán, J. C. (2018). Las buenas prácticas de enseñanza de los profesores de educación superior. REICE. Revista Iberoamericana sobre Calidad, Eficacia y Cambio en Educación, 16(2), 133-149. https://doi.org/10.15366/reice2018.16.2.008

Hallin, K. (2014). Nursing students at a university. A study about learning style preferences. Nurse Education Today, 34(12), 1443-1449. https://doi.org/10.1016/j.nedt.2014.04.001

Harrington, J. y Parker, J. (2013). Emerging technologies as cognitive tools for authentic learning. British Journal of Educational Technology, 44(4), 607-615. https://doi.org/10.1111/bjet.12048

Hernández, R., Fernández, C. y Baptista, P. (2014). Definición del alcance de la investigación que se realizará: exploratorio, descriptivo, correlacional o explicativo. En R. Hernández, C. Fernández y P. Baptista (Eds.), Metodología de la Investigación (pp. 88-101). Ciudad de México: McGraw-Hill.

Hernández-Pina, F., García, M. P., Martínez, P., Hervás, R. y Maquilón, J. (2002). Consistencia entre motivos y estrategias de aprendizaje en estudiantes universitarios. Revista de Investigación Educativa, 20(2), 487-5 10. 
Herrero, R., González, I. y Marín, V. (2015). Renovación metodológica para el desarrollo de competencias en el alumnado de educación Superior. Etic@net.Revista Científica Electrónica de Educación y Comunicación en la Sociedad del Conocimiento, 15, 109-133.

Hidalgo, N. y Murillo, F. J. (2017). Las concepciones sobre el proceso de evaluación del aprendizaje de los estudiantes. REICE. Revista Iberoamericana sobre Calidad, Eficacia y Cambio en Educación, 15(1), 107-128. https://doi.org/10.15366/reice2017.15.1.007

Hortigüela, D., Salicetti, A., Hernando, A. y Pérez, A. (2015). El trabajo autónomo del alumno universitario en las clases de Educación Física. Análisis de su percepción sobre la práctica. Ágora. Revista para la Educación Física y el Deporte, 17(3), 251-265.

Imaz, J. J. (2015). Aprendizaje basado en proyectos en los grados de pedagogía y educación social ¿Cómo ha cambiado tu ciudad? Revista Complutense de Educación, 26(3), 679-696. https://doi.org/10.5209/rev_RCED.2015.v26.n3.44665

Jansen, E. (2004). The influence of the curriculum organization on study progress in higher education. Higher Education, 47, 411-435. https://doi.org/10.1023/B:HIGH.0000020868.39084.21

Jiménez, L, García, A. J., López, J. y Saavedra, F. J. (2018). Evaluación de estrategias de aprendizaje mediante la escala ACRA abreviada para estudiantes universitarios. Revista de Psicodidáctica, 23(1), 63-69. https://doi.org/10.1016/j.psicod.2017.03.001

Kramarski, B. y Michalsky, T. (2009). Investigating preservice teachers' professional growth in self-regulated learning environments. Journal of Educational Psychology, 101(1), 161-175. https://doi.org/10.1037/a0013101

López, M. (2010). Diseño y análisis del cuestionario de estrategias de trabajo autónomo (CETA) para estudiantes universitarios. Revista de Psicodidáctica, 15(1), 77-99.

Martínez, A. (2015). Percepciones de los estudiantes sobre el proceso de convergencia europeo. European Journal of Child Development, Education and Psychopathology, 3(2), 69-79. https://doi.org/10.30552/ejpad.v3i2.30

Martínez, R. y Bonachea, O. (2011). ¿Estrategias de enseñanza o estrategias de aprendizaje? Recuperado de http://es.slideshare.net/hilderlino/estrategias-de-enseanza-o-estrategiasde-aprendizaje

Mehrdad, A. G. y Ahghar, M. (2012). Learning styles and learning strategies of left-handed EFL students. Procedia-Social and Behavioral Sciences, 31, 536-545. https://doi.org/10.1016/j.sbspro.2011.12.100

Muñoz, M. (2005). Estrategias de aprendizaje en estudiantes universitarios. Revista Electrónica de Psicología Científica, 7(11), art 1.

Olmedo, E. M. (2013). Enfoques de aprendizaje de los estudiantes y metodología docente: Evolución hacia el nuevo sistema de formación e interacción propuesta en el EEES. Revista de Investigación Educativa, 31(2), 411-429. https://doi.org/10.6018/rie.31.2.133501

Rezende, F. M., Fonseca, L. J., Nunes, V., Da Silva, G. y Antas, L. (2014). A student-centered approach for developing active learning: The construction of physical models as a teaching tool in medical physiology. BMC Medical Education, 14, 189-198. https://doi.org/10.1186/1472-6920-14-189

Richardson, J. T. (1995). Mature students in higher education. An investigation of approaches to studying and academic performance. Studies in Higher Education, 20, 5-17.

https://doi.org/10.1080/03075079512331381760 
Sadler-Smith, E. (1996). Approaches to studying: Age, gender and academic performance. Educational Studies, 22, 367-379. https://doi.org/10.1080/0305569960220306

Tejada, J. y Ruiz, C. (2016). Evaluación de competencias profesionales en educación superior: Retos e implicaciones. Educación XX1, 19(1), 17-38.

https://doi.org/10.5944/educxx 1.12175

Valle, A., Cabanach, R. G., Rodríguez, S., Núñez, J. C. y González-Pineda, J. A. (2006). Metas académicas, estrategias cognitivas y estrategias de autorregulación del estudio. Psicothema, 18, 165-170.

Vallejo, M. y Molina, J. (2011). Análisis de las metodologías activas en el grado de maestro en educación infantil: La perspectiva del alumnado. Revista Electrónica Interuniversitaria de Formación del Profesorado, 14, 207-217.

Wolters, C.A. (2004). Advancing achievement goal theory: using goals structures and goal orientations to predict students 'motivation, cognition and achievement. Journal of Educational Psychology, 96, 236-250. https://doi.org/10.1037/0022-0663.96.2.236

Zhang, L. (2000). University students learning approaches in three cultures: an investigation of Biggs`s 3P Model. The Journal of Psychology, 134(1), 37-55.

https://doi.org/10.1080/00223980009600847

\section{Breve CV de la autora}

\section{$M^{a}$ del Carmen Pegalajar}

Profesora Ayudante Doctora del Departamento de Pedagogía de la Universidad de Jaén, adscrita al área de Didáctica y Organización Escolar. Doctora por la Universidad de Jaén con la obtención de Premio Extraordinario en el ámbito de Ciencias Sociales y Jurídicas. Licenciada en Psicopedagogía y Diplomada en Maestro, especialidad en Educación Primaria. Máster en Educación Especial. Miembro del grupo de investigación "Desarrollo e Investigación de la Educación en Andalucía" de la Universidad de Jaén. Sus líneas de investigación se centran el análisis de la formación docente, la educación inclusiva y la calidad de la Educación Superior. ORCID ID: https://orcid.org/ooooo001-9408-6770. Email:mcpegala@ujaen.es 